\title{
2nd German-French DNA repair meeting - DNA damage and repair in ageing and degenerative diseases, Konstanz, Germany, September 20-23, 2009
}

\author{
Alexander Bürkle*, Alain Sarasin, Lisa Wiesmüller \\ Department of Biology, Box X911, University of Konstanz, 78457 Konstanz, Gemany \\ Laboratory of Genetic Stability and Oncogenesis, CNRS UMR8200, mstitut Gustave Roussy, University Paris 5ud, 94805 Villejuif, France \\ Department of Obstetrics and Gyncecology, University of Ulm, Pritwitzstr. 43, 89075 Ulm, Germany
}

A B S T R A C T

In September 2009, the French Society of Genetic Toxicology and the German Society for Research on DNA Repair jointly organized the '2nd German-French DNA repair meeting -. DNA damage and repair in ageing and degenerative diseases', which was held in Konstanz, Germany. Here we summarize the

Keywords:

DNA repair

DNA damage signaling

Aging content of the oral presentations given in the various scientific sessions and of prize-winning posters.

Degenerative disorders

Cancer

After the great success of the first joint scientific meeting of the French Society of Genetic Toxicology (www.sftg.org) and the German Society for Research on DNA Repair (www.dgdr.de) in Toulouse, France, in 2007 the two societies decided to jointly organize a second meeting, which was held in Konstanz, Germany, September 20-23, 2009. Although the Konstanz meeting was open to presentations of any kind of scientific activity in DNA repair research, the meeting had a focus on "DNA Damage and Repair in Ageing and Degenerative Diseases". This choice was very successful because among the 126 participants it attracted there were a good number of scientists from the field of biogerontology who had never attended previous meetings of the two societies. Furthermore, although it was organized by two national societies, participants came from many more countries, thus tuming it into an international event. These two facts fostered scientific exchange and new contacts very much. Other very positive and encouraging aspects were (i) the strong representation of female speakers, especially among the presenters of proffered papers, which had been selected by the Scientific Committee based on anonymized abstracts, and (ii) the large number and high quality of posters presented. In the following paragraphs we summarize the content of the oral presentations in the various scientific sessions.

\footnotetext{
- Corresponding author. Tel.: +497531884035 ; fax: +497531884033 E-mail address: Alexander.Buerkle@uni-konstanz.de (A. Bürkle).
}

\section{Keynote lecture and public lecture}

The keynote lecture was given by Jan Hoeijmakers (Rotterdam, The Netherlands). It was entitled "Aging, DNA repair and cell death" and covered an impressive range of experimental approaches. He started out by recapitulating that DNA damage is a classical trigger of mutations, which may lead to cancer, but can also induce cell death or senescence, which may contribute to aging. The latter is the main tenet of the DNA Damage Theory of Aging. In order to antagonize accumulation of DNA damage DNA repair systems have evolved. Hoeijmakers and colleagues have focused on nucleotide excision repair (NER), which is organized in two modes, i.e. Global Genome NER, which prevents mainly mutations (and thereby cancer) and is deficient in some forms of Xeroderma pigmentosum (XP). (ii) In contrast, TranscriptionCoupled Repair (TCR) selectively removes damage that obstructs transcription, thus counteracting cytotoxic effects of DNA injury (thereby delaying aging). Clinical examples are Cockayne syndrome (CS) and trichothiodystrophy (TTD), two syndromes featuring signs of premature aging features yet without cancer susceptibility. Strikingly, mutations in the NER helicases XPB and XPD can cause all three disorders or combinations thereof. Hoeijmakers showed that different single and double mouse NER mutants exhibit growth deficit, neurodegeneration, osteoporosis, early loss of fertility, premature aging of liver and kidney, deafness, retinal photoreceptor loss, depletion of hematopoietic stem cells, and a moderate or even dramatic reduction of life span. There was a strong correlation between the severity of repair defects and the severity of premature aging, in support of the 
Endogenous DNA damage

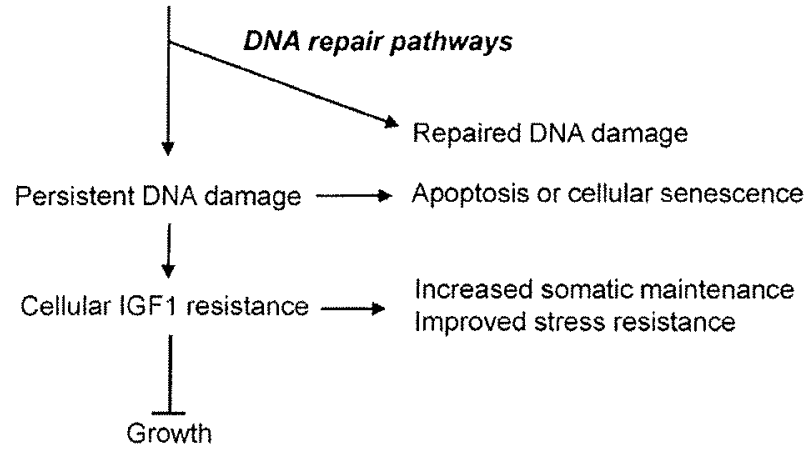

Fig. 1. Simplified scheme on the implication of unrepaired DNA damage in the aging process. Inverted T, blockage. For details, see text

DNA-damage theory of aging. Strikingly, conditional mouse mutants display organ-specific accelerated aging, including targeted neurodegeneration in specific parts of the brain. In summary, endogenous DNA lesions recognized by NER pathways can hamper transcription/replication thus triggering apoptosis and premature aging. Importantly, persisting DNA damage suppresses the somatotrophic axis and up-regulates defenses, thus favoring maintenance at the expense of growth, a phenomenon similar to caloric restriction, which is known to promote longevity in many species (Fig. 1).

The public lecture was delivered by Vilhelm A. Bohr (Baltimore, MD, USA) and was entitled "DNA repair in aging and degenerative disease". In the first part of his lecture he addressed the Mitochondrial Theory of Aging, which can be viewed as an extension to Denham Harman's Free Radical Theory of Aging. The Mitochondrial Theory suggests that the mitochondrial decline with aging is due to mitochondrial DNA damage accumulation and is causative of many of the aging features. While all oxidation of macromolecules may be important, oxidative modification of DNA may be the most harmful to the organism. Thus, removal of oxidative DNA damage is a key defense process, and, similar to the situation in the nucleus, some mitochondrial DNA repair pathways exist to keep the steady-state level of damage low. However, the classical NER, described above, is not active in mitochondria. In the second part of his presentation, Bohr discussed human progeroid syndromes as these conditions have proved to be very suitable model systems for the study of aging. These diseases include Werner syndrome (WS), Bloom syndrome, Rothmund-Thomson syndrome, CS and others. An increasing number of proteins that are deficient in human progeroid syndromes seem to play roles in DNA repair. Bohr and his colleagues focused on the Werner syndrome protein (WRN) and other RecQ helicases and characterized interactions with major DNA double-strand break repair and BER proteins. Recently, they reported a physical and functional interaction between WRN and NEIL1 glycosylase. Their current data indicate that another RecQ helicase, the Rothmund-Thomson protein (RecQ 4 ) is also involved in these DNA repair pathways. Another important protein that antagonizes premature aging is CSB. This protein also interacts with base-excision repair (BER) proteins including NEIL1.

\section{DNA repair, ageing and age-related diseases}

Hartmut Geiger (UIm, Germany) investigates DNA damage responses in the aged hematopoietic stem cell system in the mouse, in order to unravel the mechanisms underlying the well- known fact that cancer in general is a disease tightly associated with aging. He could show that hematopoietic progenitor cells, but not hematopoietic stem cells, from aged mice are more resistant to DNA damage compared to cells from young animals. Thus, the more likely cancer-initiating cell in age-related cancer could be a hematopoietic progenitor cell. Furthermore, he showed that the mutation frequency upon DNA damaging treatment does not increase in hematopoietic cells in aged animals and concluded that not only DNA mutations but distinct additional mechanisms might be the driving force in age-related cancer. A likely candidate is epigenetic modifications of DNA.

In general, all biological macromolecules including DNA, RNA, proteins, lipids and polysaccharides can undergo molecular damage, but repair activities have almost exclusively been described for DNA. The only exception is repair of a very specific kind of protein damage, i.e. oxidation of methionine residues leading to methionine sulfoxide formation, which was the topic of the presentation by Bertrand Friguet (Paris, France). Such protein damage can be reversed by the methionine sulfoxide reductase MsrA and MsrB. The Msr system has been implicated as an activity contributing to increased longevity and resistance to oxidative stress in different cell types and model organisms. Msr activity and expression of MsrA decrease with age in vivo, and both MsrA and MsrB2 are downregulated during cellular senescence. Frigutet showed that overexpression of MsrA or MsrB2 in transfected cells leads to lower levels of protein oxidative damage and protects against oxidative stress-induced cell death. Interestingly, he could show that Msr overexpression also leads to lower level of intracellular ROS under oxidative stress conditions and protected both mitochondrial integrity and proteasome function against oxidative stress-induced inactivation. Viewed together it is exciting to see how major biochemical pathways/systems that are all tightly linked to the aging process, such as mitochondria, the cellular redox balance and the proteasome, are all responsive to interventions targeted at repair of oxidative protein damage.

Bjön Schumacher (Cologne, Germany) focused on the role of persistent transcription-blocking DNA damage in the aging process. He showed that persistent DNA damage in primary cells elicits similar changes in global gene expression as those occurring in various organs of naturally aged animals. As is the case in aging animals, the insulin-like growth factor 1 (IGF-1) receptor and growth hormone receptor expression is attenuated under these conditions, resulting in cellular IGF-1 resistance. This cellautonomous attenuation is specifically induced by persistent lesions that lead to RNA polymerase II stalling. It is detectable in proliferating, quiescent and terminally differentiated cells, and is exacerbated and prolonged in cells from progeroid mice and confers resistance to oxidative stress. He concluded that DNA damage accumulation in transcribed genes contributes to the aging-associated shift from growth to somatic maintenance that triggers stress resistance and is thought to antagonize tumorigenesis and promote longevity (Fig. 1)

Sylvie Sauvaigo (Grenoble, France) described a multiplexed excision/synthesis assay to examine simultaneously BER and NER capacities. She tested human primary fibroblasts derived from healthy donors of various ages and investigated changes in DNA repair attributed to chronic sun exposure, by comparing fibroblasts from sun-exposed and sun-protected sites of the same donors. She observed a marked age-related decrease of excision/synthesis repair whatever the repair pathway considered. Interestingly enough, distinct decline profiles were observed according to the nature of the base lesion studied. Cells from middle-age donors exhibited sun-exposure related adaptive up-regulation of repair activity of 8-oxoguanine and abasic sites. By contrast, cells from sun-exposed sites of elderly donors displayed significantly lower repair capacity than cells from sun-protected sites for these latter 
lesions, which is indicative of an additional adverse effect of sunlight exposure, apart from direct induction of DNA damage.

Miriam Uht from the group led by Lisa Wiesmüller (Ulm, Germany) presented recent data on the involvement of the class III histone deacetylase (HDAC) SIRT1 in homology-directed DNA double-strand break (DSB) repair in human cells. Nuclear foci analysis and application of pathway-specific, fluorescence-based, chromosomal DSB reporters in combination with pharmacological or siRNA-mediated inactivation of SIRT1 revealed activation of single-strand annealing (SSA) in particular. Among the repairrelated acetylation substrates of SIRT1, the Werner syndrome protein (WRN) was found to be required for this activity, thereby providing evidence for a functional link between these two antiaging proteins.

Nadine Miebach from the group of Christian Rïbe (Homburg/ Saar, Germany) reported on the accumulation of DSBs in adult tissue-specific stem cells with aging in mice. She and her colleagues quantified DSBs in epithelial stem cells and differentiated epithelial cells from skin biopsies of repair-proficient C57BL6 mice, ranging in age from 2 weeks to 24 months. The number of unrepaired DSBs was analyzed in CD34 positive epithelial stem cells compared to differentiated epithelial cells by enumerating 53BP1-foci. Unrepaired DSBs in the epithelial stem cells increased significantly with age and reached maximal values by 18 and 24 months. In contrast, only a slight age-dependent increase of DSBs was observed in the short-lived, differentiated epithelial cells. In summary, the analysis of tissue-specific stem cells within their physiological niche suggests that endogenous DNA damage accumulates over time and thus may trigger cellular senescence causing the functional decline of tissue stem cells within mammalian ageing.

Alexander Bürkle (Konstanz, Germany) presented the design of the EU-funded Large-Scale Integrating Project MARK-AGE (www.mark-age.eu) he is coordinating. The goal of this project is the identification of a robust set of biomarkers of human aging which, as a combination of parameters with appropriate weighting, would measure biological age better than any such marker in isolation. Among the large number of candidate biomarkers being systematically studied in a population of 3700 subjects recruited at 8 different places across Europe are BER function (by automated FADU assay) and cellular poly(ADP-ribosyl)ation capacity. Preliminary data point to a decrease of these two parameters with chronological age. In addition telomere length and epigenetic stability are also being analyzed.

\section{DNA damage signaling}

Françoise Dantzer (Strasbourg, France) presented data on the cooperative and specific functions of three members of the family of poly(ADP-ribose) polymerases (PARPs), ie. PARP-1, PARP-2 and PARP-3. PARPs catalyze poly(ADP-ribosyl)ation of proteins, and PARP-1 and PARP-2 have specifically been shown to play an essential role in genome integrity and were described as active players in the base-excision repair/single-strand break recognition and repair processes (BER/SSBR). However, Dantzer and colleagues recently identified a splice variant of human hPARP3, lacking exon 1, as another nuclear, DNA-dependent PARP, whereas the fulllength enzyme has been previously described as a core component of the centrosome. Dantzer and colleagues found hPARP-3 to associate with Polycomb group protein complexes involved in transcriptional silencing and with DNA repair networks of BER/ SSBR and non-homologous end-joining. By laser microirradiation and live cell microscopy, the group identified recruitment of PARP. 3 to localized DNA damage sites. Using immunoprecipitation and mass spectrometry analysis, proteins involved in mitotic progression were identified to be PARP-3 interactors in human cell extracts. Collectively the data suggest an active role for PARP-3 in the maintenance of genomic integrity.

In her lecture, Valeria Naim from the group led by Filippo Rosselli (Villejuif, France) presented an interesting facet of the links between DNA repair and chromosome segregation. Together with her colleagues, she discovered that the DSB repair factors FANCD2 and FANCI localize to non-centromeric discrete sites on metaphase chromosomes when resolution of stalled replication forks fails. She convincingly showed that the proteins, which are mutated in Fanconi anemia (FA) and Bloom's syndrome (BLM), are necessary to avoid chromosome breakage or loss during replication stress caused by converging forks, secondary structures, or chromosome entanglement. Fragile sites are particularly prone to this type of defective anaphase separation, which attributes a critical role of the FA pathway in the maintenance of stability of fragile sites.

Thomas von Zglinicki (Newcastle upon Tyne, UK) presented data on the dynamics of DNA damage foci in cell cycle and senescence. DSBs induce the formation of DNA damage, foci which contain activated ATM/ATR, YH2AX, 53BP1 and other proteins and are necessary for the induction and execution of a DNA-damage response, leading to DNA repair, permanent cell cycle arrest (cell senescence) or apoptosis. Von Zglinicki and colleagues used a 53BP1-GFP fusion protein as a reporter for focus formation in living cells. He showed that during mitosis, $\gamma \mathrm{H} 2 \mathrm{AX}$ foci present in $S$ phase can remain attached to chromosomes, but 53BP1 is removed from foci in $\mathrm{G} 2$ and remains completely absent from chromosomes during metaphase. He also showed that only some DNA damage foci typically observed in senescent cells are potentially permanent, while upto $50 \%$ of all foci in senescing human fibroblasts are transient and are continuously replenished. This is due to the fact that the DNA damage response in senescing cells induces mitochondrial dysfunction and production of reactive oxygen species (ROS) via signaling through p21 (CDKN1A), p38 MAPK and TGFB. DNA damage response and mitochondrial ROS production thus form a positive feed-back loop that is necessary and sufficient to maintain permanent growth arrest until the full senescent phenotype is established.

Heinz-Peter Nasheuer (Galway, Ireland) focused on the human single-stranded DNA-binding protein, RPA, as a component of damage-induced signaling during mitosis. He identified several $\mathrm{N}$ terminal sites in the $32 \mathrm{kDa}-\mathrm{RPA} 2$ subunit, which are rapidly hyperphosphorylated in response to DNA damage during $M$-phase. Radiation-induced phosphorylation during mitosis turned out to rely on ATM and to a lesser extent on DNA-PK, but not ATR, and changed the localization of RPA from chromatin-excluded to chromatin-associated, suggesting participation in DSB repair during mitosis.

Beare Volkmer (Buxtehude, Germany) reported on epigenetic changes induced by UV-radiation. UV-light is a well-known mutagen and inducer of skin cancer, but in the past few years evidence has been emerging that epigenetic modifications, especially in epidermal stem cells, are also of great importance for skin carcinogenesis, and that $\mathrm{CpG}$ island promoter hypermethylation and global genomic hypomethylation are hallmarks of (skin-) cancer. In addition, post-translational changes of histones act in concert with DNA methylation in order to regulate gene expression. Using methylation-specific PCR, chromatin immunoprecipitation, immunofluorescence microscopy and flow cytometry Volkmer and colleagues were able to show that UV-radiation indeed leads to promoter hypermethylation in a number of genes known to be involved in the genesis of skin cancer as well as $\mathrm{H} 3 \mathrm{~K} 9$ and $\mathrm{H} 3 \mathrm{~K} 27$ patterns.

The heterogeneous group of recessive progressive ataxias was the subject of the presentation by Michel Koenig (Strasbourg, France). This group of neurodegenerative disorders can be caused 
by various defects in genes coding for mitochondrial, cytoplasmic, and nuclear proteins. All these proteins are never found to be completely absent, except for ATM, whose functions are thought to be compensated by ATR. The nuclear proteins ATM (ataxiatelangiectasia, AT) and MRE11 (ataxia-telangiectasia-like disorder, ATLD) are involved in DSB repair. Aprataxin (ataxia with oculomotor apraxia 1, AOA1) removes DNA adenylates from aborted ligation reactions during $S S B$ repair and BER; TDP1 (spinocerebellar ataxia with axonal neuropathy 1, SCAN1) resolves topoisomerase I intermediates. As an exception among the nuclear proteins, senataxin ( $A O A 2$ ) plays no role in DNA repair, but seems to act in transcription termination. Thus, it appears that neuronal cells are particularly sensitive to a number of specific lesions.

Ralph Gruber from the group of Zhao-Qj Wang (Jena, Germany) studied the mechanism by which MCPH1 maintains mammalian brain size. MCPH1 is a regulator of the DNA damage response; it forms nuclear foci after ionizing or UV irradiation, which colocalize with 53BP1, MDC1, NBS1, ATM, RPA, and ATR. MCPH1 regulates transcription of Chk1 and BRCA1 or their stability. MCPH1 mutations cause Primary Microcephaly (MCPH), a neurodevelopmental disorder characterized by reduced brain size, mental retardation and, at the cellular level, premature chromosome condensation (PCC). Gruber and colleagues disrupted the Mcph1 gene in mice, which led to microcephaly due to neocortical developmental defects and increased apoptosis in the ventricular and subventricular zones of the neocortex. Neural progenitors proliferate normally in Mcph1-deficient mice but show a severely constrained self-renewal capacity, causing premature cell cycle exit. Further, the group showed that deletion of Mcphl affects the division mode of neuroprogenitors by promoting asymmetric cell division and neuronal fate.

\section{Telomeres}

Eric Gilson's (Lyon, France) work is focused on the shelterin complex, and especially how TRF 2 promotes t-loop formation by a single-strand invasion mechanism and protects Holliday junctions from resolvase attack, thereby coping with the problem of telomeric replication fork arrest and consequent telomere shortening. TRF2 is known to modulate several repair pathways as already suggested by its promiscuous interactions with proteins such as Apollo, BLM, WRN, and PARP1/2. Focusing on the most recently identified binding partner Apollo, a $5^{\prime}$-exonuclease, he showed that expression of a nuclease-dead allele elicits a DNA damage response at telomeres in S-phase. In vitro experiments demonstrated that TRF2 stimulates the $S^{\prime}$-exonuclease activity of Apollo on single-stranded DNA and inhibits the same activity on double-stranded DNA. This finding motivated FISH analyses that revealed that TRF2 and Apollo protect interstitial telomeric DNA sequences in particular. Gilson proposed a model according to which TRF2 couples the exonuclease activity of Apollo to replication in order to eliminate clastogenic and recombinogenic structures and to prevent recombination at replication-linked break sites within the inner part of telomeres.

K. Lenhard Rudolph (UIm, Germany) addressed the role of DNA damage in adult stem cell aging and organ maintenance in the context of telomere dysfunction. There is growing evidence that aging of adult stem cells contributes to age-associated defects in organ maintenance and to cancer development, and telomere shortening emerges as one of the underlying mechanisms contributing to stem cell aging. Rudolph and colleagues recently analyzed the functional consequences of conditional p53 deletion in the intestinal epithelium of aging mice with dysfunctional telomeres. Intestine-specific deletion of p53 shortened the lifespan of telomere-dysfunctional mice without inducing tumor formation. In contrast to what the group had previously observed with p21 deletion, deletion of p53 impairs the clearance of chromosomally instable stem cells in the intestine of aging telomeredysfunctional mice. Such genetically instable stem cells contribute to epithelial regeneration thereby leading to an accumulation of chromosomal instability, increased apoptosis, altered epithelial cell differentiation and premature intestinal failure.

\section{Double-strand break repair}

Bernard Salles (Toulouse, France) presented one of several talks on the molecular links between telomeres and DSB repair as exemplified best by the interactions of the classical NonHomologous End-Joining (NHEJ) components $\mathrm{Ku}$ and DNA-PK with telomeres. To understand the mechanisms underlying chromosome end fusions in the absence of Ku alone or any NHEJ components together with the telomeric complex TRF2/RAP1, he set up an in vitro NHEJ assay by mixing human cell extracts and plasmid DNA bearing long telomeric duplex repeats close to the end. The results showed that $3^{\prime}$ but not $5^{\prime}$ telomeric DNA was refractory to joining, inhibited DNA-PK activity, and was competitively bound by Ku and TRF2/RAP1. Immunodepletion of TRF2/ RAP1 enabled NHEJ mediated by the classical pathway, removal of $\mathrm{Ku}$ by the alternative pathway, indicating the existence of a double protection against telomere fusions.

Martin Digweed (Berlin, Germany) systematically analyzed the consequences of altering the nibrin (NBN) status in vivo. Mice with homozygous null mutations of NBN in their livers were treated with 4 Cy $y$-radiation and subjected to proteome analysis of about 8000 liver proteins. Highly significant expression changes were found in groups of proteins involved in the oxidative stress response and cellular redox homeostasis. This finding indicated that the extremely high cancer risk associated with hypomorphic NBN mutations in Nijmegen Breakage Syndrome (NBS) patients is the result of a compound defect in DSB repair and oxidative stress response. From his recent data, he also proposed a proteasomebased rather than nonsense-mediated mRNA decay mechanism to underlie expression variations of the truncated p70-nibrin gene product of NBN c.657del5, which is the mutation found in $90 \%$ of NBS patients. This mechanism may modify the disease phenotype and has major implications for future clinical applications.

Immunodeficiency is one of the hallmarks of NBS. However, due to its essential function in cell viability, the physiological function of nibrin in $V(D)]$ recombination and lymphoid homeostasis remained elusive. Through the generation of mice with deletion of NBN in the T cell lineage, Zhao-Qi Wang (Jena, Germany) was able to show that nibrin is required for the resection and repair of DNA ends in the TCR $\beta$ and TCR $\alpha$ loci and proper RAG-mediated postcleavage complex formation, leading to deletions and insertions at $V(D)]$ coding ends and genomic instability in $N B N^{\text {T-del }}$ mice. Despite the severe lymphopenia observed early in life, the mice developed an autoimmune pathology after 12 months of age due to depletion of regulatory $T$ cells, revealing an interesting mechanistic link between immunodeficiency and autoimmunity.

Barbara Koch and Olivia Barton presented recent work by the group of Markus Löbrich (Darmstadt, Germany). Koch summarized the data obtained with EdU/BrdU/PCNA staining, phophoH3/ $\gamma H 2 A X$ co-staining, life cell imaging with the break marker GFP. 53BP1 and the S-phase marker pDSRed-Ligasel, as well as PCC testing for chromosomal breaks that altogether revealed that cells are released into mitosis with 10-20 unrepaired DSBs. In contrast, the $\mathrm{G} 1 / \mathrm{S}$ checkpoint is thought to be initiated even in the presence of a single DSB. However, she observed that the G1/Scheckpoint is strong but slowly activated, so that single cells escape G1 arrest after irradiation and enter $S$ - and G2-phase. Olivia Barton compared DSB repair activities in G1- and G2-phases by CENP. $\mathrm{F} / \gamma \mathrm{H} 2 \mathrm{AX}$ co-staining and analyzed the consequences of inactivat- 
ing different DSB repair components. Her data showed that BRCA2driven Homologous Recombination, but not the classical NHE] pathway, contributes to the slow DSB repair phase in G2 Retinoblastoma-binding protein 8 (CtIP) depletion accelerated repair by switching from $\mathrm{HR}$ to NHEJ. Most interestingly, ATM and Artemis turned out to be required for the slow repair phases in $C 1$ and $\mathrm{C} 2$, but only in the presence of CIIP. ATM-activated Artemis was, therefore, postulated to play a role in removing secondary end structures that may arise from the resection by CtIP.

Christian Mielke (Düsseldorf, Germany) applied a laser line with a wave-length of $364 \mathrm{~nm}$ combined with live cell imaging of GFP$53 \mathrm{BP} 1$ fusion proteins to investigate the influence of UVA on the genomic integrity. Following irradiation at physiological doses, GFP-53BP1-foci labeled sites were marked by $\gamma \mathrm{H} 2 \mathrm{AX}$ suggesting DSBs formation. Since foci were not restricted to the irradiated regions and since treatment with an anti-oxidant reduced signals at these distant sites, he concluded that a diffusible molecular intermediate, such as ROS, can mediate significant DSB formation after UVA-irradiation.

The experiments described by Claudia Rübe (Homburg/Saar, Germany) were inspired by the fact that the main function of germ line stem cells is to maintain genome integrity in order to prevent reproduction failure and to limit hereditary risk upon transmission to progeny. Consequently, she compared radiation-induced DSB repair in PLZF-positive spermatogonial stem cells (SSCs) versus differentiated A and B spermatogonia, spermatocytes, and spermatids. Examination of $\gamma \mathrm{H} 2 \mathrm{AX}-, 53 \mathrm{BP} 1-, \mathrm{Mdc1}-$, and Rad51foci showed clearly higher DSB repair efficiency in SSCs compared to all other spermatogenic cells. This interesting finding is in apparent contrast to the generally reduced DSB repair efficiency described for male germ cells compared to somatic tissue-cells.

Marie Dutreix (Orsay, France) reported on hyperactivation of DNA-PK by double-strand break-mimicking molecules and the ensuing disturbance of the cellular DNA damage response. She and her colleagues designed short and stabilized double-stranded DNA molecules (Dbait) mimicking double-strand breaks. Dbait molecules with a length $\leq 24$-bp bind Ku proteins but not DNA-PKcs, whereas longer versions bind the whole DNA-PK complex and activate its kinase activity. By contrast, ATM is not activated by these molecules. Activation of DNA-PK induces a phosphorylation cascade that remains activated for several days in the cell but is insufficient to cause cell cycle arrest or apoptosis. Irradiation of cells with Dbait-activated DNA-PKcs restores cell cycle arrest and apoptosis, but does not allow organization of repair proteins in foci at the damage site. Prior administration of Dbait molecules sensitizes radioresistant tumor xenografts to ionizing irradiation.

Thorsten Rieckmann from the group of Jochen Dahm-Dahpi (Hamburg, Germany) re-evaluated the role of p53 in homologous recombination (HR). To initiate DSB repair at different time points during the cell cycle, he and his colleagues transfected synchronized DSB repair reporter cells with a tamoxifen-inducible I-Scel expression plasmid and subsequently added tamoxifen at different time points. In this way he found that HR starts to repair DSBs after early S-phase. In agreement with the observations made previously by other groups, he also demonstrated that expression of wild-type 533 in monkey cells endogenously expressing wild-type p53 repressed HR in S- and G2-phase; tumor-derived mutant p53 caused the opposite response.

\section{Excision repair}

Vincent Laugel (Strasbourg, France) gave an overview on clinical and genetic features of CS and cerebro-oculo-facio-skeletal (COFS) syndromes. CS is an autosomal recessive multi-system disorder characterized by progressive growth failure, microcephaly, mental retardation, retinal degeneration, sensorineural deafness and photosensitivity and is linked to mutations in the CSB/ERCC6 and CSA/ERCC8 genes which encode proteins involved in the transcription-coupled DNA repair pathway (TCR). The clinical spectrum of CS encompasses a wide range of severity. In classical type I CS, the first symptoms usually appear from the end of the first year of life, and death occurs before the end of the second decade. Early-onset cases with a more unfavorable prognosis (type II) and late-onset cases with milder symptoms (type III) have also been described. Even the very severe COFS syndrome can be regarded as a prenatal form of CS. On the other hand, the very mild UV-sensitive syndrome, which was investigated in the first French patient in Alain Sarasin's laboratory (Villejuif, France), is characterized by cutaneous photosensitivity only, without any associated neurological involvement or growth defect. At the cellular level, it shares the UV sensitivity with CS, but not the ROS sensitivity, and broadens the phenotypic spectrum of CSA and CSB mutations. Laugel and colleagues have identified 43 new mutations in CSA and CSB genes in addition to the $\mathbf{4 5}$ known mutations. Many types of mutations are scattered along the whole coding sequence of the two genes, but clusters of missense mutations can be recognized and highlight the role of particular motifs in the proteins.

In his lecture Jean-Marc Egly (Strasbourg, France) gave a comprehensive overview of the molecular components of the TFIH complex, which fulfills a dual function in transcription and NER. In particular, using his elaborate assays that fully reconstitute biochemical processes with the individual, highly purified components, he was able to dissect these activities by showing that XPE functions as an ATP-driven hook to stabilize the binding of TFIIH to damaged DNA, whereas XPD represents the transcriptionspecific TFIIH helicase subunit. Moreover, he identified the kinase CAK as the molecular component that switches TFIH activities from transcription to repair, thereby drawing a more complete picture of the orchestrating role of this caretaker complex.

The knowledge on how NER acts on unusual DNA structures, such as regions with high capacity for base unwinding, is still rather limited. $\$ 23906$ is an antitumor alkaloid drug forming bulky monofunctional adducts with a unique capacity to destabilize local base pairing. Amnette $K$. Larsen (Paris, France) reported that cells deficient for XPC, CSB or XPA are profoundly sensitive to $\$ 23906$. In contrast, cells deficient in ERCC1 and XPF, which are involved in later steps of NER, showed only modest sensitivity to \$23906. In addition Larsen and colleagues developed S23906-resistant cells by continued exposure to increasing concentrations of $\$ 23906$ in growth medium. This was accompanied by cross-resistance to cisplatin and UV irradiation as well as by increased NER activity. Resistant cells show upto five-fold upregulation of XPC, CSB and XPA proteins while ERCC1 levels were unchanged.

Within the global genome NER repair pathway, the XPC protein is a key general sensor of helix-distorting DNA lesions arising from UV-radiation, genotoxic chemicals and reactive metabolic byproducts. Flurina C. Clement from the group of Hanspeter Naegeli (Zurich, Switzerland) presented data on a two-stage dynamic DNA quality check by XPC. She and her colleagues monitored the realtime redistribution of fluorescent XPC fusion proteins following high-resolution DNA damage induction. Bidirectional truncation analyses disclosed a surprisingly short recognition hotspot in human XPC that includes two $\beta$-hairpin domains, with a preference for non-hydrogen-bonded bases in double-stranded DNA. Additional data revealed a two-stage discrimination process whereby XPC protein first deploys a dynamic sensor interface to rapidly interrogate the double helix, thus forming a transient recognition intermediate before the final installation of a more static repair-initiating complex.

The crystal structure of XPD from Thermoplasma acidophilum was presented by Stefanie $C$. Wolski from the group of Caroline 
Kisker (Würzburg, Germany). She showed that the protein consists of two RecA-like helicase domains, a 4 Fe 45 cluster, and an $\alpha$ helical domain, which form a ring with a diameter of $13 \hat{A}$, i.e. sufficient in size to allow passage of single-stranded DNA. She proposed that the damage-containing DNA strand passes through this central hole and is checked for damaged bases. This model rationalizes the positions of several mutations that lead to the diseases XP, CS or TTD.

Thomas lingg from the group of Bernd Epe (Mainz, Germany) demonstrated the detrimental effect of DNA single-strand breaks (SSBs) on gene expression by use of a GFP-based reporter after NB.Bpu10I- or Nt.BpulOI-mediated nicking in the transcribed and non-transcribed strand, respectively. In CSB-deficient cells the effect on transcription was further exacerbated, however, for SSBs in the non-transcribed strand. From this and SSB repair measurements in chromosomal DNA by alkaline elution, he concluded that CSB is not important for global SSB break repair but necessary for transcription of damaged genes.

Error-prone DNA repair of AID-induced damage is the mechanism underlying somatic hypermutation in B cells, whereas error-free $\mathrm{HR}$ is necessary for IgG gene conversion. Berit Jungnickel (Munich, Germany) reported on a shift from conservative errorfree to non-conservative error-prone HR in constitutively hypermutating human germinal centre B cell lines. Based on the analysis of DT 40 cell lines with targeted inactivation of candidate genes, she demonstrated that Rad18 from the Rad6 pathway, which leads to ubiquitination of PCNA, was critical for the suppression of errorfree repair during somatic hypermutation. Ubc13, which is involved in PCNA poly-ubiquitination, was required for $\lg G$ gene conversion, but somatic mutation was unaffected.

Martina Bauer from the group of Bernd Kaina (Mainz, Germany) studied the sensitivity of primary monocytes, dendritic cells (DCs), and macrophages to oxidative stress that can arise spontaneously, during inflammatory responses, and after exposure to exogenous agents. After treatment with tert-butylhydroperoxide, monocytes but not DCs or macrophages, were found to be sensitive to apoptosis, which was correlated with a defect in BER and decreased expression of the BER proteins PARP1, XRCC1, and Ligase III. These data showed that monocytes are vulnerable to oxidative damage before maturation into DCs and macrophages, which could impair the immune response after chemotherapeutic treatment.

Prediction of the responsiveness to dacarbazine or temozolomide treatment of melanoma was the subject of the talk given by Lars Boeckmam from the group of Stefan Emmert (Gottingen, Germany). The efficacy of treatment with these methylating agents depends on low repair by MGMT and high mismatch repair by the host. mRNA expression, genetic variants, and the promoter methylation status of MGMT, MSH2, and MLH1 were determined in a study on patients with metastasizing melanoma. None of these parameters served to significantly predict treatment response, except for the genetic MSH2 variant rs 2303428 . This report calls for future studies involving functional assays.

\section{Mitochondria}

Mathis Mueller-Ohldach, from the group of Heinz D. Osiewacz (Frankfurt am Main, Germany), focused on mitochondrial DNA repair and aging in the filamentous fungus Podospora anserina, a well-established model organism of aging, with a lifespan of about 20 days. Since it is well established that mitochondrial DNA stability and respiration are pivotal in the control of the aging process in $P$. anserina, the group compared BER processes in mitochondria from young and old fungi. A significant, agedependent drop in BER activity against four damage substrates was detectable. Furthermore an in silico search for BER-associated proteins identified four candidates of high homology to known BER factors. The group is currently testing the effects of overexpressing the respective proteins targeted to mitochondria, which should compensate for the loss of activity during aging. It will be interesting to see if reactivating the mitochondrial BER has any influence on age-related cellular changes in this organism.

\section{Poster sessions}

As mentioned above, the large number and high quality of posters presented were certainly a highlight of the meeting, and a total of four were selected for poster prizes. These posters are summarized below:

Noma Baum (from the group of Frank Grosse, Jena, Germany) described that p 53 interacts physically and functionally with the peptidyl-prolyl cis/trans isomerase cyclophilin 18 (Cyp18). The group observed that Cyp18 was found to bind to the proline-rich region of $\mathrm{p} 53$ via the active site of this cis/trans isomerase. Cyp 18 binding decreased the sequence-specific binding activity of p53 in vitro and Cyp 18 inhibited the transcriptional activity of a p53reporter gene in vivo. The $C y p 18$-inhibitor cyclosporin $A$ induced a p53-dependent cell cycle arrest in cultured cells. Furthermore, genetic ablation of cyp18 led to an increased p53-mediated apoptotic response, suggesting an anti-apoptotic potential of Cyp18-p53 complexes.

forg Fahrer (from the group of Holger Barth, Ulm, Germany) reported on C2IN-streptavidin, a novel cell-permeable transport system for mammalian cells and its potential use to modulate DNA repair and genomic stability. $\mathrm{C} 2 \mathrm{IN}$, which is the $\mathrm{N}$-terminal domain of the Clostridium botulinum toxin component $\mathrm{C} 2 \mathrm{I}$, is important for C2lla-mediated internalization of $\mathrm{C} 2 \mathrm{I}$. A recombinant $\mathrm{C} 2 \mathrm{IN}$ streptavidin fusion protein was constructed enabling delivery of exogenous biotinylated macromolecules into mammalian cells. Using an overlay blot technique, a biotinylated protein was shown to bind in a concentration-dependent manner to C2IN-streptavidin. Internalization of C2IN-streptavidin into the cytosol of Vero cells was monitored by immunoblot analysis and confocal microscopy. Biotin-fluorescein was used as model substance for this novel delivery system. Further studies are underway to assess the transport of biotinylated proteins such as the tumor suppressor p53.

Aswin Mangerich (from the group of Alexander Bürkle, Konstanz, Germany) presented data on mice with ectopic gene targeting of human poly(ADP-ribose) polymerase-1 (hPARP-1). Due to the dual role of PARP-1 in cellular mechanisms such as genomic maintenance on the one hand and cell death and inflammation on the other hand, this enzyme is discussed both as a longevity factor and as an aging-promoting factor. Since hPARP-1 exhibits a significantly enhanced poly(ADP-ribosyl)ation capacity compared to its rodent (rat) or tholog the group generated and characterized a novel mouse model with ectopic expression of hPARP-1, hPARP-1-expressing mice displayed impaired survival rates in, with signs of premature aging. This phenotype might be related to a continuous low-level increase in pro-inflammatory stimuli, potentially due to altered PARP-1-NFKB interaction, consistent with the paradigm of 'inflammaging".

Sabrina Köcher (from the group of Jochen Dahm-Daphi, Hamburg, Germany) performed a comparative study on ATMand Artemis-deficient cells. The radiosensitive syndromes Ataxia teleangiectasia (AT) and Artemis both show a severe defect in NHE] but only ATM-deficient cells display attenuated G1/S and G2/M checkpoints. The group asked whether the differences in cell cycle regulation are coupled to deficient homologous recombination (HR) repair of DSBs, and they could show that upon X-irradiation of exponentially growing cells, both Artemis and AT cells showed a substantial accumulation in the G2-phase. To test if this was due to activation of ATR by S-phase related DSB, cells were exposed to 
hydrogen peroxide at concentrations that only induced SSB and base damage. Only AT cells accumulated in $\mathrm{C} 2$, indicating that passage through $\mathrm{S}$-phase is necessary to induce a G2-checkpoint in AT cells.

\section{Perspectives}

After the great success of the first two French-German DNA repair meetings, it was decided to continue this series and to organize a third one, probably in Strasbourg (France) at the end of 2011 or beginning of 2012. All possible efforts will be made to make this forthcoming meeting a big success, too. In particular young researchers will encouraged to participate, to make new contacts, and to engage in trans-national cooperation. One of the final goals of this endeavor is to facilitate exchange between laboratories both in terms of ideas and science, but also in terms of students and post-doctoral fellows.

\section{Acknowledgement}

The financial contribution by the Deutsche Forschungsgemeinschaft towards the organization of the meeting (under grant BU 698/7-1) is gratefully acknowledged. 